\author{
Volume 8. No. 5, May 2020
}

International Journal of Emerging Trends in Engineering Research

Available Online at http://www.warse.org/IJETER/static/pdf/file/ijeter61852020.pdf

https://doi.org/10.30534/ijeter/2020/61852020

\title{
Design of Cascaded Adaptive Filter for ECG Denoising Applications
}

\author{
M.Chitra $^{1}$, S.Sasikala ${ }^{2}$, S.Gomathi ${ }^{3}$, A.Neetheswaran ${ }^{4}$, C.Reetha ${ }^{5}$ \\ ${ }_{1,2,3}$ Assistant Professor, ${ }^{4,5}$ Students \\ Department of Electronics \& Communication Engineering, Kongu Engineering College, Perundurai, India. \\ chitra.ece@kongu.edu, sasikalas@kongu.ac.in,samgomathi@gmail.com
}

\begin{abstract}
Two stage LMS adaptive filter is designed for the purpose of ECG noise cancellation. The output of first LMS filter is given as input for second LMS. Error signal of first LMS filter is given as desired signal for second LMS filter. Signal to noise ratio of single LMS filter is compared with cascaded LMS adaptive filter and it is analyzed that noise elimination is more in cascaded LMS adaptive filter. The SNR value for 8-tap LMS filter using cascaded technique is $21.52 \%$ higher than the normal 8-tap LMS filter structure. The SNR value for 16-tap LMS filter using cascaded technique is $24.84 \%$ higher than the normal 16-tap LMS filter structure.
\end{abstract}

Key words: Adaptive filter, Cascaded LMS, Signal-to-noise-ratio, ECG.

\section{INTRODUCTION}

Adaptive filter is a self-modifying filter its frequency response to change its behavior in time. For minimizing error it is used. The error function is distance between the reference or desired signal and the output of the adaptive filter. Mean squared error between primary input (and the reference input is reduced by the adaptive filter .LMS is based on gradient descent algorithm in which instantaneous error signal is used to update the filter coefficients. For removing or suppressing noise from signal this technique is used.

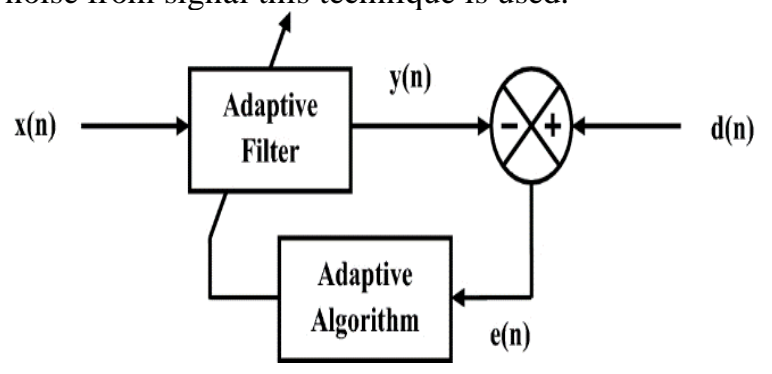

Figure 1: LMS Adaptive filter

Figure 1 shows the structure of LMS adaptive filter. Responses of noise cancellation in terms of signal-to-noise ratio with respect to filter lengths are determined. The algorithm for the first LMS filter is described as desired signal of an adaptive filter is $d(n)$, error signal of an adaptive filter is $\mathrm{e}(\mathrm{n})$, output of an adaptive filter is $\mathrm{y}(\mathrm{n})$,step size of an adaptive filter is $\mu$, filter weight vector of an adaptive filter is $\mathrm{w}(\mathrm{n})$, input signal vector $\mathrm{x}(\mathrm{n})$ and filter length is Primary input and reference input are the two input sources. The primary input source receives the desired signal (signal with noise) will include source signal. Signal got corrupted. y(n) generated the reference input signal which generated same source. This output produce e(n) error signal equivalent to noise source.

\section{RELATED WORKS}

Systolic and folded architectures are combined in different adaptive filters like RLS, Kalman and Affine Projection filters [1]. A New approach for noise cancellation in speech signal using affine projection algorithm for attenuating noise in speech signals [2]. Adaptive Filter algorithms based on Adaptive Noise Cancellation (ANC) systems is provided in order to reduce computational complexity for future implementation and to increase the convergence rate [3]. Two stage LMS noise cancellation technique is used for improving signal to noise ratio [4]. Adaptive RLS filter is used for denoising speech signals [5]. A system that can perform the WBCs, RBCs and platelets count from the blood sample taken by caretakers is developed [7]. Presented Implementation of Least Mean Square (LMS) algorithm using two different architectures. Results shows floating point implementation to perform well than other methods [8].

\section{METHODOLOGY}

In adaptive filtering, the Least mean square adaptive filter is used many applications. Various techniques are available for cascading adaptive filters. The proposed method is the Adaptive noise cancellation by means of Cascaded LMS Adaptive filter method. Cascaded adaptive LMS filter combination performs better noise cancellation when compared to single adaptive filter. There are many adaptive algorithms like RLS, Weiner filter, Kalman filter, but the commonly used is the LMS algorithm. Cascaded LMS adaptive filter has improved Signal-to-noise when compared to single LMS adaptive filter. There is a reduction in noise level in the signal when cascaded feedback adaptive LMS 
filter is used. The block diagram of cascaded Least Mean Square adaptive filter is shown in figure 2.

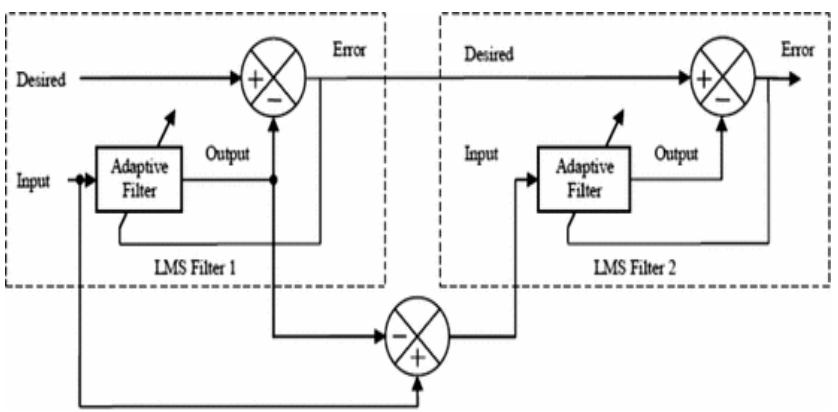

Figure 2: Cascaded LMS Adaptive Filter

Figure 2 shows the structure of cascaded LMS adaptive filter. The desired input for first LMS adaptive filter is ECG signal and the input signal is The parameters used for simulation of second LMS filter are $\mathrm{dC}(\mathrm{n})$ the cascaded desired signal, the cascaded LMS error signal is $\mathrm{eC}(\mathrm{n})$, the cascaded output of LMS adaptive filter is $\mathrm{yC}(\mathrm{n})$, the step size of cascaded LMS adaptive filter is $\mu \mathrm{c}$ is, the filter weight vector of cascaded LMS adaptive filter is $\mathrm{WC}(\mathrm{n})$ is, $\mathrm{XC}(\mathrm{n})$ is the cascaded input signal vector and $\mathrm{L}$ is the cascaded LMS filter length. The signal-to-noise ratio of second stage adaptive filter is higher than the first stage signal-to-noise ratio of adaptive filter.

\section{RESULTS}

The filters were designed with 4-tap, 8-tap, 16-tap and 32tap length and the Signal-to-Noise Ratio (SNR) was calculated for all the tap length.

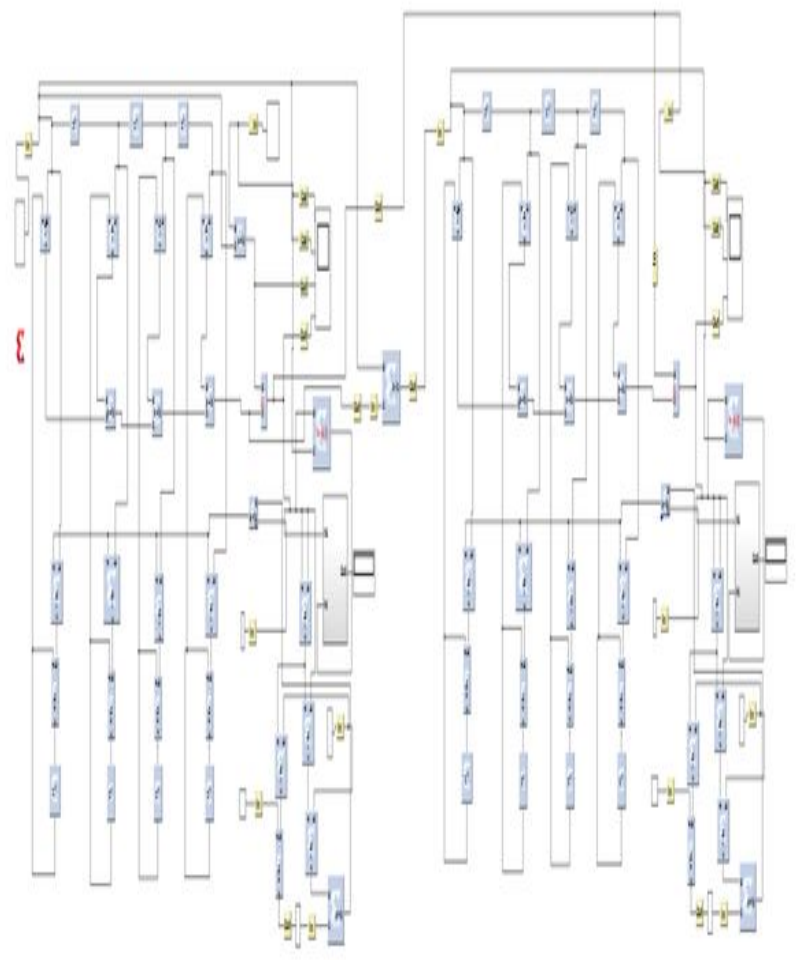

Figure 3: 4 Tap Cascaded LMS adaptive filter
Figure 3 shows the design of four tap cascaded LMS adaptive filter structure in System Generator. The contaminated ECG signal is the input to the filter and PLI noise is the reference input signal to the filter.

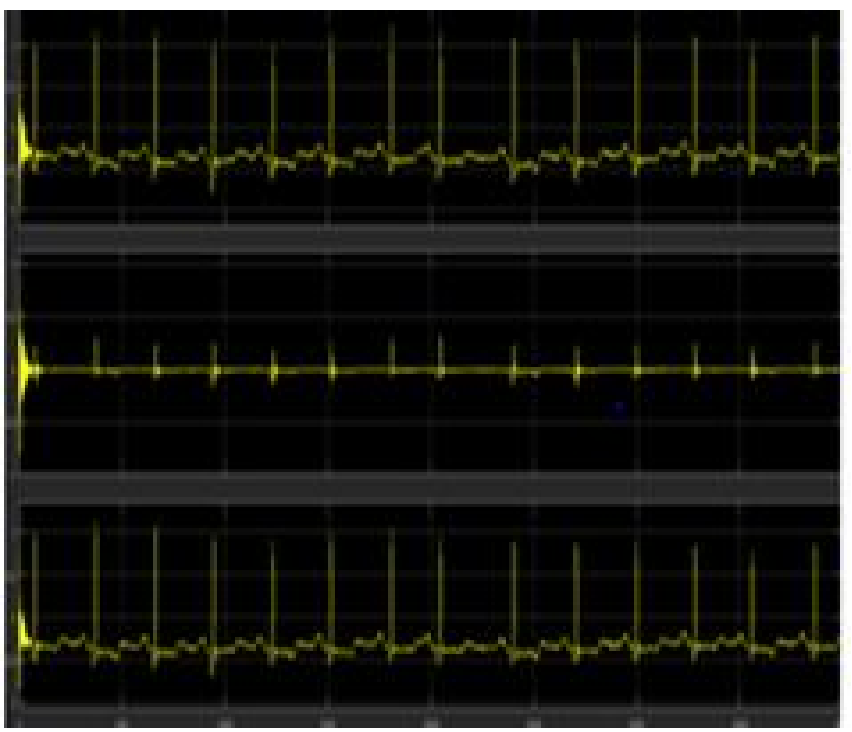

Figure 4: Simulation output of 4 - tap LMS cascaded adaptive filter structure in system generator

Figure 4 shows the denoised signal is obtained, after passing the ECG signal added with PLI noise to the four tap cascaded LMS filter. Subtract the filtered output from desired input in order to get original ECG signal.

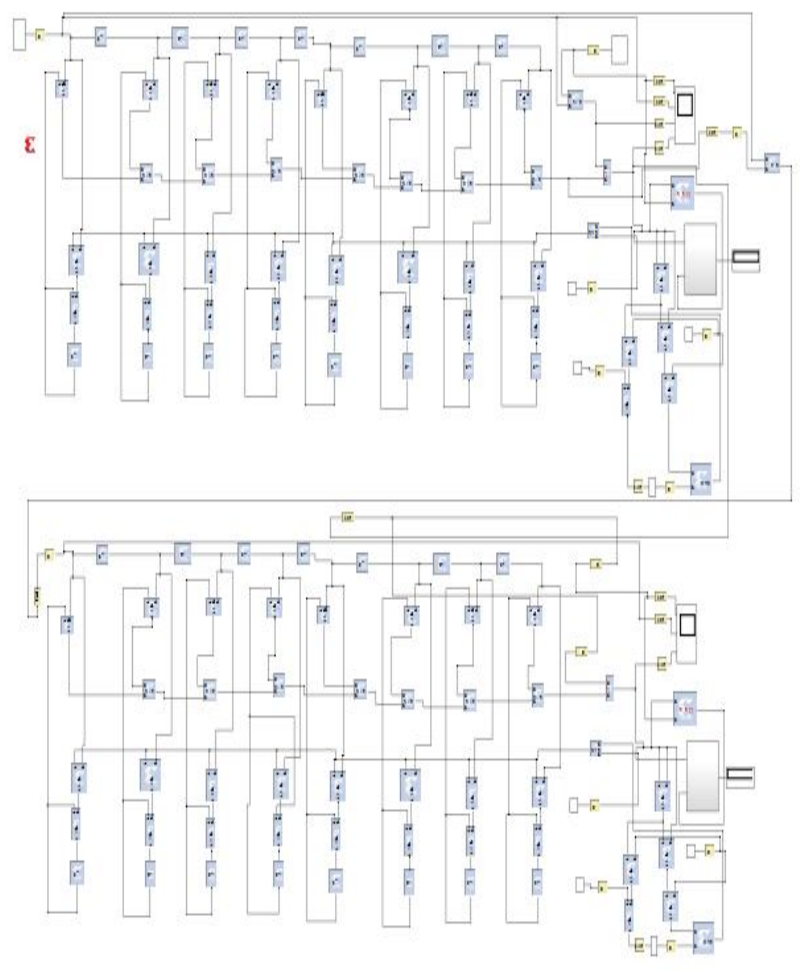

Figure 5: 8 - tap cascaded LMS adaptive filter structure in system generator 
Figure 5 Shows the design of eight tap cascaded LMS adaptive filter structure I system generator. The contaminated ECG signal is the input to the filter and PLI noise is the reference input signal to the filter

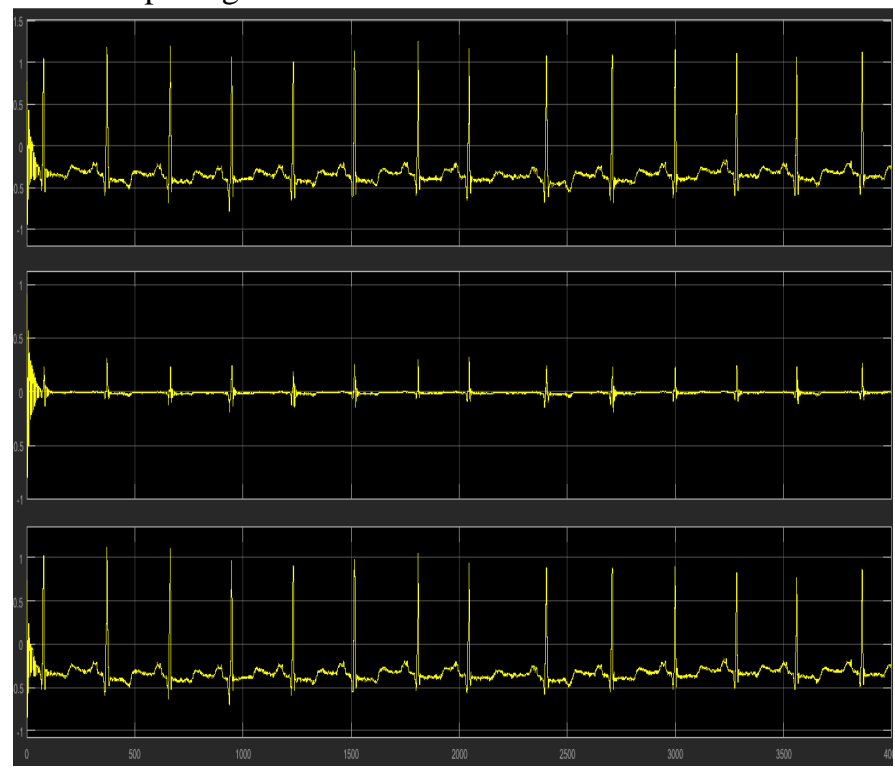

Figure 6: Simulation output of 8 - tap cascaded LMS adaptive filter structure in system generator

In Figure 6, the denoised signal is obtained, after passing the ECG signal added with PLI noise to the eight tap cascaded LMS filter using high level transformation techniques .Subtract the filtered output from desired input in order to get original ECG signal.

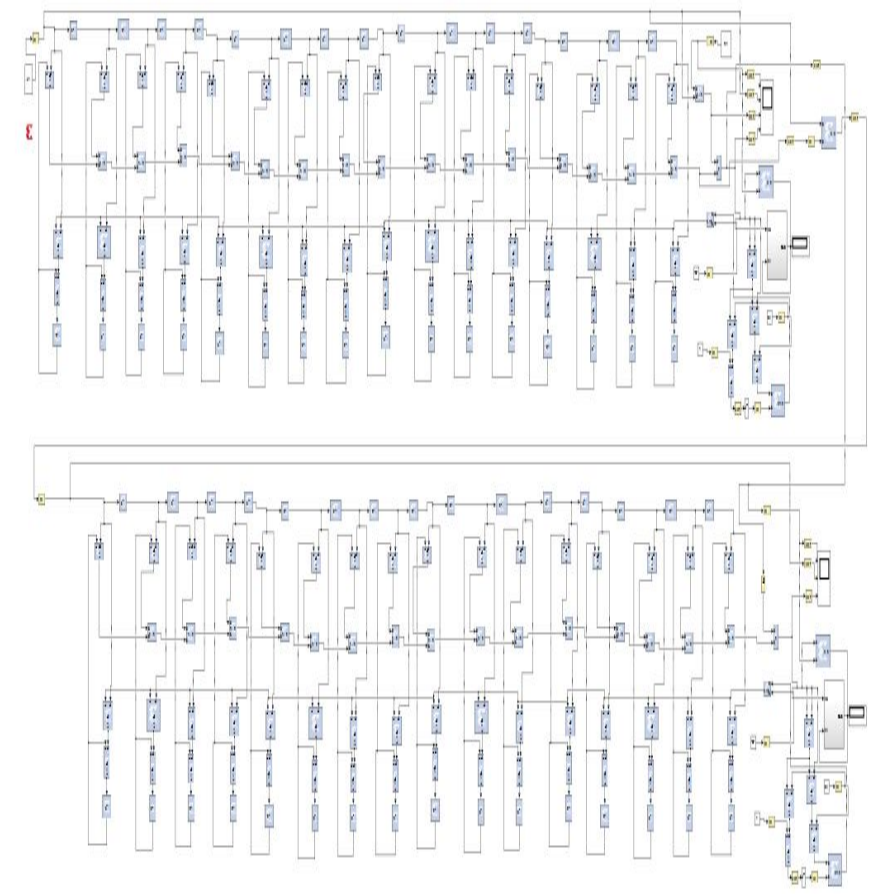

Figure 7: 16 - tap cascaded LMS adaptive filter structure in system generator
Figure 7 Shows the design of 16 tap cascaded LMS adaptive filter structure in System Generator. The contaminated ECG signal is the input to the filter and PLI noise is the reference input signal to the filter.

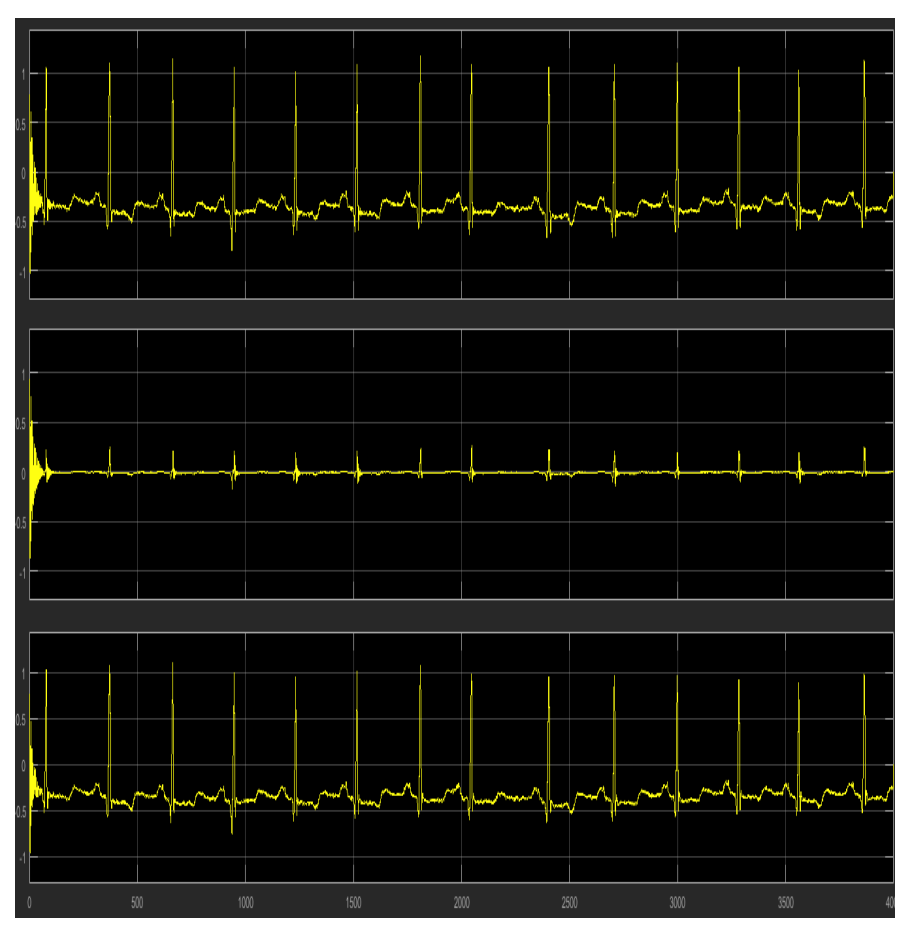

Figure 8: Simulation output of 16 - tap cascaded LMS adaptive filter structure in system generator

In Figure 8, The denoised signal is obtained, after passing the ECG signal added with PLI noise to the 16 tap cascaded LMS filter. Subtract the filtered output from desired input in order to get original ECG signal.

Table 1 shows comparison of signal to noise ratio of ECG signals with PLI noise for 4-tap LMS, 8-tap LMS and 16-tap LMS adaptive filters with and without using cascaded techniques. From the table it is found that the SNR value for 4-tap LMS filter using cascaded technique is $24.54 \%$ higher than the normal 4-tap LMS filter structure. The SNR value for 8-tap LMS filter using cascaded technique is $21.52 \%$ higher than the normal 8-tap LMS filter structure. The SNR value for 16-tap LMS filter using cascaded technique is $24.84 \%$ higher than the normal 16-tap LMS filter structure. 
M.Chitra et al., International Journal of Emerging Trends in Engineering Research, 8(5), May 2020, 1850- 1853

Table 1: Comparison of SNR for LMS and Cascaded LMS Adaptive Filters

\begin{tabular}{|l|l|l|l|l|l|l|}
\hline \multirow{2}{*}{$\begin{array}{l}\text { Input } \\
\text { ECG } \\
\text { Signal }\end{array}$} & LMS & $\begin{array}{l}\text { Length of the filter -4 } \\
\text { Cascaded } \\
\text { LMS }\end{array}$ & LMS & $\begin{array}{l}\text { Length of the filter -8 } \\
\text { Cascaded } \\
\text { LMS }\end{array}$ & \multicolumn{2}{l|}{ Length of the filter -16 } \\
\hline 100.dat & 30.99 & 34.15 & 37.77 & 40.08 & 37.16 & $\begin{array}{l}\text { Cascaded } \\
\text { LMS }\end{array}$ \\
\hline 101.dat & 31.83 & 39.59 & 30.24 & 48.67 & 37.36 & 41.06 \\
\hline 105.dat & 29.34 & 32.49 & 31.2 & 34.1 & 35.79 & 40.71 \\
\hline 108.dat & 28.96 & 34.48 & 28.28 & 30.79 & 34.05 & 43.34 \\
\hline 200.dat & 24.93 & 31.64 & 27.77 & 33.51 & 27.79 & 33.69 \\
\hline 203.dat & 21.07 & 35.2 & 24.68 & 31.28 & 26.91 & 33.41 \\
\hline 208.dat & 30.4 & 34.71 & 29.94 & 41.24 & 24.3 & 30.19 \\
\hline 228.dat & 30.79 & 42.08 & 38.58 & 42.25 & 37.36 & 50.13 \\
\hline
\end{tabular}

\section{CONCLUSION}

This paper presented the design of Cascaded RLS adaptive filter for denoising ECG signal. Xilinx System generator is used for designing structures. The SNR value for 8-tap LMS filter using cascaded technique is $21.52 \%$ higher than the normal 8-tap LMS filter structure. The SNR value for 16-tap LMS filter using cascaded technique is $24.84 \%$ higher than the normal 16-tap LMS filter structure. From the analysis cascaded structure performs better noise cancellation.

\section{REFERENCES}

1. Swaminathan.G, Murugesan.G, Sasikala.S, Murali.L. A novel implementation of combined systolic and folded architectures for adaptive filters in FPGA Microprocessors and Microsystems, no.74, 2020. https://doi.org/10.1016/j.micpro.2020.103018

2. Satyanarayana.J and Amjadkhan.G. Affine Projection Algorithm Applied to Adaptive Noise Cancellation, International Journal of Electronics and Communication Technology, IJECT, vol.3, no.1, March 2012.

3. Shubhra Dixit.S, Deepak Nagaria. Design and analysis of cascaded LMS adaptive filters for noise cancellation, Circuits, Systems and Signal Processing 36(2), 742-766, 2017.

https://doi.org/10.1007/s00034-016-0332-5
4. Awadhesh Kumar Maurya. Cascade Cascade Least Mean Square (LMS) Adaptive Noise Cancellation, Circuits, Systems and Signal Processing, DOI: 10.1007/s00034-017-0731-2, 2017.

5. Ahmad.S, Ahmad.T. Implementation of Recursive Least Squares (RLS) Adaptive Filter for Noise Cancellation, International Journal of Scientific Engineering and Technology, vol.1, pp. 46-48, 2012.

6. Vishal Chandra, Prattay Guha Sarkar, Vinay Singh. Mitral Valve Abnormality Detection Using Deep Learning Approach in PLAX view in TTE, International Journal of Emerging Trends in Engineering Research, vol.8, no. 4, April 2020.

7. Kantilal Rane. Online Rpi-Web-Server based Blood Cell Analysis for Fast Diagnosis and Monitoring of Disorders for Remote Stations, International Journal of Emerging Trends in Engineering Research, vol.8, no. 4, April 2020.

8. Shashikala Prakash, Renjith Kumar T.G, Subramani.H. An FPGA Implementation Of The LMS Adaptive Filter for Active Vibration Control, International Journal of Research in Engineering and Technology, vol.2, no. 10, 2013.

https://doi.org/10.15623/ijret.2013.0210001 